\title{
Cardiac syncope in a woman with undiagnosed celiac disease
}

\author{
Laïla Samy MD, Alan Barkun MD MSc, Thao Huynh MD PhD
}

Cite as: CMAJ 2017 March 6;189:E365-7. doi: 10.1503/cmaj.151043

A 42-year-old white woman presented to the emergency department with a one-week history of exertional nausea, dizziness and syncope. She reported no infectious symptoms and had no chest pain, palpitations, dyspnea, orthopnea or paroxysmal nocturnal dyspnea. She had no gastrointestinal symptoms other than nausea preceding the syncopal episodes.

The patient had a history of mild childhood asthma and two uncomplicated vaginal deliveries. She was not taking medications, had no allergies and did not consume recreational drugs or alcohol. Her family history was remarkable only for a cousin with a bicuspid aortic valve. Specifically, there was no family history of celiac disease or other autoimmune conditions. The patient had travelled to the Rocky Mountains five months before presentation but had not noticed tick bites.

Findings on physical examination were normal. The first electrocardiogram (ECG) at rest showed a right bundle branch block with first-degree atrioventricular block (Figure 1). Laboratory investigation showed normal results for complete blood count, electrolytes, and renal and thyroid function (Appendix 1, available at www.cmaj.ca/lookup/suppl/doi:10.1503/cmaj.151043/-/ DC1). Troponin levels, C-reactive protein level, results of an autoimmune workup (including anti-extractable nuclear antigen, rheumatoid factor and anti-DNA antibody), angiotensinconverting enzyme level, complement levels and immunoglobulin profile were normal. The result of serologic testing for Lyme disease was also negative.

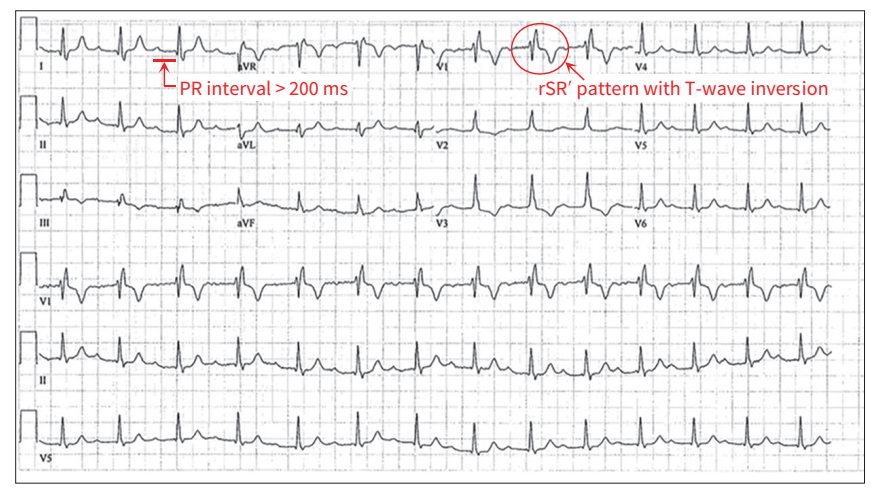

Figure 1: Electrocardiogram on presentation showing right bundle branch block with first-degree atrioventricular block.

\section{KEY POINTS}

- Celiac disease can present with cardiac disease without accompanying gastrointestinal symptoms.

- Celiac disease should be considered in young patients with unexplained dilated cardiomyopathy, myocarditis or conduction disease.

- A gluten-free diet may improve cardiac symptoms in patients with celiac disease and concomitant cardiac disease.

An exercise stress test induced symptomatic (dizziness and nausea) alternating right and left bundle branch blocks and several premature ventricular complexes. Results of echocardiography and coronary angiography were normal. Although cardiac magnetic resonance imaging (MRI) scans were suggestive of possible myocarditis (septal edema was observed), a gallium scan did not show abnormal cardiac uptake of radioisotopes.

An iron profile, undertaken to exclude cardiac hemochromatosis, suggested iron deficiency, with a ferritin level of 6 (normal 11-306) $\mu \mathrm{g} / \mathrm{L}$, despite a normal hemoglobin level of 126 (normal 120-160) g/L. Testing for tissue transglutaminase (tTG) antibodies, done as part of the iron deficiency workup, yielded elevated levels (tTG immunoglobulin $\mathrm{A}>200 \mathrm{U} / \mathrm{mL}$ ). A gastroscopy was done and showed scalloping of the duodenal folds (Figure 2). Duodenal biopsy confirmed a diagnosis of celiac disease, showing markedly increased intraepithelial lymphocytes and partial villous atrophy of the duodenal mucosa, which is consistent with Marsh type 3 a celiac disease ${ }^{1}$ (Figure 2 ).
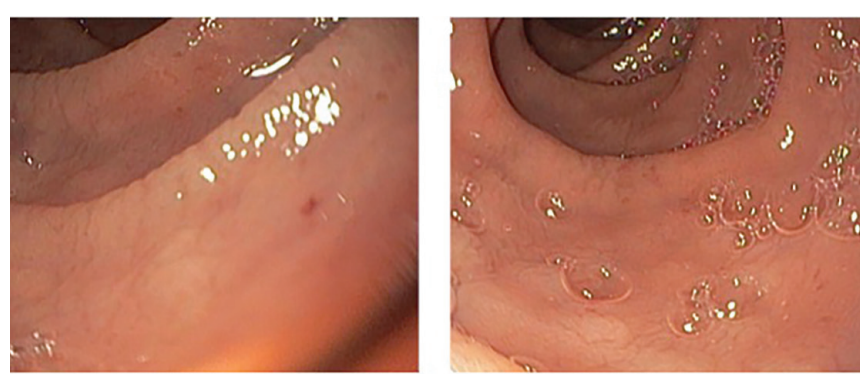

Figure 2: Duodenal mucosa on initial gastroscopy showing scalloping of the duodenal folds. 


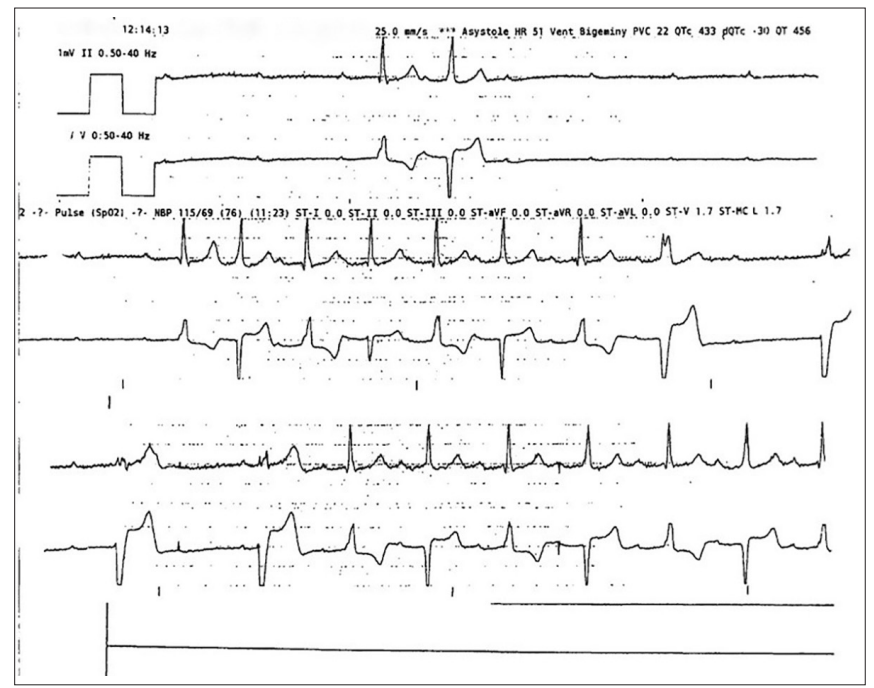

Figure 3: Findings from cardiac monitor after minimal exertion, showing prolonged pause with high-grade atrioventricular block.

While in hospital, the patient's cardiac monitor showed a highly symptomatic six-second pause with high-grade atrioventricular block (Figure 3) after minimal walking. A pacemaker was subsequently inserted because of the increased frequencies of spontaneous alternating bundle branch blocks and prolonged sinus pauses.

After confirmation of celiac disease on duodenal biopsy, the patient started following a gluten-free diet. She experienced almost immediate resolution of her symptoms. Pacemaker interrogation showed 99\% ventricular pacing before and 3.5\% ventricular pacing five months after she started the gluten-free diet. The patient's resting ECG also returned to normal (Appendix 2, available at www. cmaj.ca/lookup/suppl/doi:10.1503/cmaj.151043/-/DC1).

\section{Discussion}

Our patient presented with syncope secondary to conduction disease, which required pacemaker insertion. The differential diagnosis of conduction disease in her age group includes coronary artery disease, exaggerated vagal tone, medications, nonischemic cardiomyopathies and infectious causes (e.g., viral myocarditis and Lyme disease). Other possibilities include rheumatic and autoimmune disorders (e.g., systemic lupus erythematosus, giant cell myocarditis, ankylosing spondylitis and rheumatoid arthritis), infiltrative processes (e.g., amyloidosis, sarcoidosis, non-Hodgkin lymphoma and multiple myeloma) and degenerative diseases (e.g., Lenègre and Lev diseases). ${ }^{1}$

There are no current guidelines for the investigation of conduction disease in healthy young or middle-aged adults. The basic investigation of such patients typically includes measurement of cardiac enzyme levels and of inflammatory and autoimmune markers, as well as echocardiography and MRI to exclude cardiac infiltrative disease. The results of these tests were normal in our patient, apart from iron deficiency, which led us to investigate the possibility of celiac disease.

The patient's conduction abnormalities resolved after she began following a gluten-free diet. Although hemochromatosis is clearly associated with infiltrative cardiac disease that can lead to arrhythmia, there are no data suggesting that iron deficiency alone without anemia could be related to cardiac disease. Although there is some evidence in the literature that suggests an association between celiac disease and cardiac disease, we cannot ascribe causality between celiac disease and the conduction disorder in our patient, but rather a temporal association.

\section{Celiac disease}

Celiac disease is an immune-mediated malabsorptive disorder that occurs in about $1 \%$ of the general population and $5 \%-20 \%$ of their first-degree relatives. ${ }^{2}$ Classic manifestations include recurrent diarrhea, weight loss, abdominal distention, iron-deficiency anemia and unexplained nutritional deficiencies (e.g., of fatsoluble vitamins, vitamin $B_{12}$, folate and iron). ${ }^{2}$ Screening for celiac disease is recommended only in symptomatic individuals or first-degree relatives according to the 2013 American College of Gastroenterology guideline. ${ }^{2}$

Patients with celiac disease usually have positive IgA anti-tTG antibody levels; however, concomitant IgA deficiency can lead to false-negative results. In this situation, the American College of Gastroenterology guideline recommends measuring anti-tTG IgG levels or anti-deamidated gliadin peptide IgG antibody levels. Patients with a high probability of celiac disease or positive serologic markers should undergo a small-bowel biopsy for diagnostic confirmation. ${ }^{2}$ These investigations should be completed without gluten restriction.

\section{Cardiac conditions and celiac disease}

Although uncommon, several types of cardiac involvement have been reported in association with celiac disease. These include dilated cardiomyopathy, myocarditis, pericarditis and conduction disease.

As shown in two large population-based cohort studies, patients with celiac disease are at increased risk of idiopathic dilated cardiomyopathy (hazard ratio [HR] 1.73, 95\% confidence interval [Cl] 1.0-3.0) $)^{3}$ and atrial fibrillation (HR 1.34, 95\% Cl 1.24-1.44). ${ }^{4}$ In a cross-sectional study, Frustaci and colleagues ${ }^{5}$ found biopsyconfirmed celiac disease in $4.4 \%$ of 187 patients with myocarditis, as compared with $0.3 \%$ in the control group $(p<0.003)$. All nine patients had iron deficiency anemia without gastrointestinal complaints, and all had substantial improvement of cardiac symptoms after starting a gluten-free diet.

Rare cardiac manifestations of celiac disease in adults have been described in case reports and include pericardial effusion ${ }^{6}$ and pericarditis. ${ }^{7}$ Cases of heart block associated with celiac disease have been reported, but they were associated with other contributing conditions. In two separate case reports, patients were found to have atrioventricular block in the presence of both celiac disease and idiopathic pulmonary hemosiderosis., ${ }^{8,9}$ The authors of these reports suggested that the cardiac conduction disease was related to pulmonary hemosiderosis rather than to celiac disease. Dilated cardiomyopathy was a concomitant diagnosis noted in two other patients with celiac disease presenting with heart block..$^{10,11}$

Several explanations for cardiac involvement in celiac disease have been proposed. These include nutritional deficiencies, abnor- 
mal intestinal permeability increasing the absorption of luminal antigens, and a direct autoimmune reaction targeting antigens present in both the myocardium and the small intestine. ${ }^{12}$

A gluten-free diet is the mainstay of treatment in classic celiac disease. Patients usually experience near total resolution of gastrointestinal symptoms within weeks to months. In patients presenting with dilated cardiomyopathy associated with celiac disease, a small observational study documented improvement of left ventricular function in two of three patients after 28 months of adherence to a gluten-free diet. ${ }^{13}$ However, in the case reports described earlier, a gluten-free diet did not improve the conduction disease in patients with celiac disease and dilated cardiomyopathy or heart block; the patients all required insertion of a permanent pacemaker at 12 to 22 months' follow-up. ${ }^{8-11}$

\section{Conclusion}

We report a challenging case of a 42-year-old woman presenting with high-grade heart block. Subsequent investigations revealed the presence of celiac disease, which was successfully treated, followed by almost complete resolution of her conduction disease. Although further study is needed to confidently establish a causal relation between celiac disease and conduction disease, clinicians should be aware of the association between celiac disease and cardiac diseases such as dilated cardiomyopathy or unexplained arrhythmia.

\section{References}

1. Barra SN, Providencia R, Paiva L, et al. A review on advanced atrioventricular block in young or middle-aged adults. Pacing Clin Electrophysiol 2012;35: 1395-405.

2. Rubio-Tapia A, Hill ID, Kelly CP, et al. ACG clinical guidelines: diagnosis and management of celiac disease. Am J Gastroenterol 2013;108:656-76, quiz 77.

3. Emilsson L, Andersson B, Elfstrom P, et al. Risk of idiopathic dilated cardiomyopathy in 29000 patients with celiac disease. J Am Heart Assoc 2012;1: e001594.

4. Emilsson L, Smith JG, West J, et al. Increased risk of atrial fibrillation in patients with coeliac disease: a nationwide cohort study. Eur Heart J 2011;32:2430-7.

5. Frustaci A, Cuoco L, Chimenti C, et al. Celiac disease associated with autoimmune myocarditis. Circulation 2002;105:2611-8.

6. Ashrafi F, Darakhshandeh A, Heidarpour M, et al. Pericardial effusion in celiac disease. Int J Prev Med 2014;5:356-9.

7. Laine LA, Holt KM. Recurrent pericarditis and celiac disease. JAMA 1984; 252:3168.

8. Mah MW, Priel IE, Humen DP, et al. Idiopathic pulmonary hemosiderosis, complete heart block and celiac disease. Can J Cardiol 1989;5:191-4.

9. Cottin V, Clerici G, Fabien N, et al. Celiac disease revealed by diffuse alveolar hemorrhage and heart block. Respir Med Extra 2006;2:89-91.

10. Callejas Rubio JL, Ortego N, Diez-Ruiz A, et al. Celiac disease presenting as chronic anemia associated with heart block. Am J Gastroenterol 1998;93:1391-2.

11. Ouali S, Chabrak S, Larbi N, et al. Dilated cardiomyopathy and atrio-ventricular block in coeliac disease. Two case reports [article in French]. Arch Mal Coeur Vaiss 2006;99:1252-5.

12. Romagnoli E, Boldrini E, Pietrangelo A. Association between celiac disease and idiopathic dilated cardiomyopathy: a case report. Intern Emerg Med 2011;6: 125-8.

13. Curione M, Barbato M, Viola F, et al. Idiopathic dilated cardiomyopathy associated with coeliac disease: the effect of a gluten-free diet on cardiac performance. Dig Liver Dis 2002;34:866-9.

\section{Competing interests: None declared.}

This article has been peer reviewed.

The authors have obtained patient consent.

Affiliations: Internal Medicine Residency Program (Samy), Division of Gastroenterology (Barkun) and Division of Cardiology (Huynh), McGill University Heath Centre, Montréal, Que.

Contributors: All of the authors contributed to the literature review. Lailla Samy was responsible for the conception of the article, completed the literature review, compiled the figures and references, and drafted the manuscript. Thao Huynh was the primary supervisor of this work. Alan Barkun revised the manuscript and provided feedback on the figures of the gastroscopy. All of the authors approved the final version to be published and agreed to act as guarantors of the work.

Acknowledgement: The authors acknowledge Dr. Alexios Hadjis for his assistance in reviewing the pacemaker's recording.

Correspondence to: Laïla Samy, laila.samy@mail.mcgill.ca 